\title{
Selection combining for noncoherent decode-and-forward relay networks
}

\author{
Ha X Nguyen ${ }^{*}$ and Ha H Nguyen
}

\begin{abstract}
This paper studies a new decode-and-forward relaying scheme for a cooperative wireless network composed of one source, $K$ relays, and one destination and with binary frequency-shift keying modulation. A single threshold is employed to select retransmitting relays as follows: a relay retransmits to the destination if its decision variable is larger than the threshold; otherwise, it remains silent. The destination then performs selection combining for the detection of transmitted information. The average end-to-end bit-error-rate (BER) is analytically determined in a closed-form expression. Based on the derived BER, the problem of choosing an optimal threshold or jointly optimal threshold and power allocation to minimize the end-to-end BER is also investigated. Both analytical and simulation results reveal that the obtained optimal threshold scheme or jointly optimal threshold and power-allocation scheme can significantly improve the BER performance compared to a previously proposed scheme.
\end{abstract}

Keywords: cooperative diversity, frequency-shift keying, fading channel, decode-and-forward protocol, selection combining, power allocation

\section{Introduction}

Cooperative diversity has recently emerged as a promising technique to combat fading experienced in wireless transmissions. The basic idea behind this technique is that a source node cooperates with other nodes (or relays) in the network to form a virtual multiple antenna system [1-7], hence providing spatial diversity. Amplifyand-forward (AF) and decode-and-forward (DF) are two well-known protocols to realize cooperative diversity. In $\mathrm{AF}$, the relays amplify and forward the received signals to the destination. In DF, the received signal at each relay node is first decoded, and then remodulated and retransmitted. Unlike the AF protocol, it is not simple to provide cooperative diversity with the DF protocol. This is due to possible retransmission of erroneously decoded bits of the message by the relays in the DF protocol $[1,4,8,9]$.

On the other hand, the issue of how to efficiently combine multiple received signals at the receiver is of practical interest and has been intensively studied, both in point-to-point and relay communication systems. Typical combining schemes include maximal ratio

\footnotetext{
* Correspondence: hxn201@mail.usask.ca

Department of Electrical and Computer Engineering, University of Saskatchewan 57 Campus Drive, Saskatoon, SK S7N 5A9, Canada
}

combining (MRC), equal gain combining (EGC), and selection combining (SC). Since SC processes only one of the received signals, it is the simplest when compared to other combining schemes [10]. In fact, SC scheme has been widely investigated for coherent DF cooperative systems in which a perfect knowledge of channel state information (CSI) is available at the receivers (at relays and destination) [11-14]. Moreover, the SC technique is especially suitable in noncoherent communications because instead of selecting the largest signal-tonoise ratio as in coherent systems, the signal branch with the largest signal-plus-noise power can be selected. Due to these advantages, the SC scheme for binary noncoherent frequency-shift keying (FSK) in point-to-point communications has also been well studied in the literature [15-18].

The majority of research works in wireless relay networks is for coherent communications. Since obtaining the channel state information (CSI) in coherent communications might be unrealistic in fast fading environment and in multiple-relay networks, there have been some recent works that exploit noncoherent modulation and demodulation in cooperative networks [19-25]. In what follows, related works and the contributions of this paper are described. 


\subsection{Related works}

Differential phase-shift keying (DPSK) has been studied for both AF and DF protocols in [19-22]. However, with the DF protocol in [20], the authors considered an ideal case that the relay is able to know exactly whether each decoded symbol is correct. The works in [21,22] examine a very simple cooperative system with one source, one relay, and one destination node. Optimal resource allocation has been studied for noncoherent systems in $[23,24]$ to further improve the error performance of the system when DPSK is employed.

A framework of noncoherent cooperative relaying for the DF protocol employing FSK has been studied in [25] in which the maximum likelihood (ML) demodulation was developed to detect the signals at the destination. Due to the nonlinearity form and high complexity of the ML scheme, a suboptimal piecewise-linear (PL) scheme was also proposed in [25] and shown to perform very close to the ML scheme. It is noted, however, that a closed-form BER approximation for either the ML or PL scheme in [25] is not readily available for networks with more than two relays. Furthermore, the BER performance with either ML or PL demodulation can still suffer from the error propagation phenomenon [6].

To address the issue of error propagation and inspired by the work in [6], reference [26] examines an adaptive noncoherent relaying scheme in which two thresholds are employed at the relays and destination as follows. One threshold is used to select retransmitting relays: a relay retransmits to the destination if its decision variable is larger than the threshold, otherwise it remains silent. The other threshold is used at the destination for detection: the destination marks a relay as a retransmitting relay if the decision variable corresponding to the relay is larger than the threshold, otherwise, the destination marks it as a silent relay. Then, the destination simply combines (in a ML sense) the signals from the retransmitting relays and the signal from the source to make the final decision. Numerical results in [26] show that, with optimal threshold values, the cooperative relaying scheme proposed in [26] can significantly improve the error performance over the schemes in [25]. Unfortunately, closed-form BER expressions are only available for the single-relay and two-relay networks in [26]. As such, the important task of optimizing the threshold values has to rely on numerical search for networks with more than two relays.

\subsection{Contributions}

This paper is also concerned with a threshold-based relaying scheme for noncoherent DF cooperative networks in which binary FSK (BFSK) is employed at the source and relays. The transmission protocol considered is as follows. After receiving the signal from the source in the first phase, each relay decides to retransmit the decoded information if its decision variable is higher than a threshold. Otherwise, it remains silent in the second phase. At the destination, selection combining is employed to select the "strongest" received signal to decode.

It should be pointed out that one practical aspect of the proposed scheme is that the destination has no information on whether a particular relay retransmits or remains silent in the second phase. This means that the destination does not known whether a received signal is from a retransmitting relay or a silent relay. Therefore, the destination might select a signal from the relay that remains silent to decode. However, this possibility happens with a very small probability due to the selection rule implemented at the destination.

The main difference between the protocol in this paper and the one in [26] is that no threshold is needed and selection combining is performed at the destination. This simpler protocol (as compared to the protocol in [26]) also allows one to obtain a closed-form BER expression for a general network with $K$ relays. This leads to a convenient optimization of threshold and power allocation among $K$ relays. Numerical results show that our BER expression is accurate. Moreover, our proposed protocol provides a superior performance under all channel conditions with similar complexity compared to the piecewise-linear (PL) receiver in [25].

\subsection{Organization of the paper}

The remainder of this paper is organized as follows. Section 2 describes the system model. Section 3 presents the BER computation and discusses how to find the optimal threshold and power allocation. Numerical and simulation results are presented in Section 4. Finally, Section 5 concludes the paper.

\section{System model}

Consider a wireless communication system in which the source node sends its message to the destination node through $K$ relay nodes. All nodes operate in a halfduplex mode, i.e., a node cannot transmit and receive simultaneously and DF protocol is employed at the relays. We consider that the relays retransmit signals to the destination in orthogonal channels ${ }^{\mathrm{a}}$ and there is no direct link between the source and destination. For convenience, the source, relays, and destination are denoted and indexed by node 0 , node $i, i=1, \ldots, K$, and node $K+1$, respectively.

Signal transmission from the source to destination is completed in two phases as illustrated in Figure 1. In the first phase, the source broadcasts a BFSK signal and the baseband received signals at node $i, i=1, \ldots, K$, are written as 


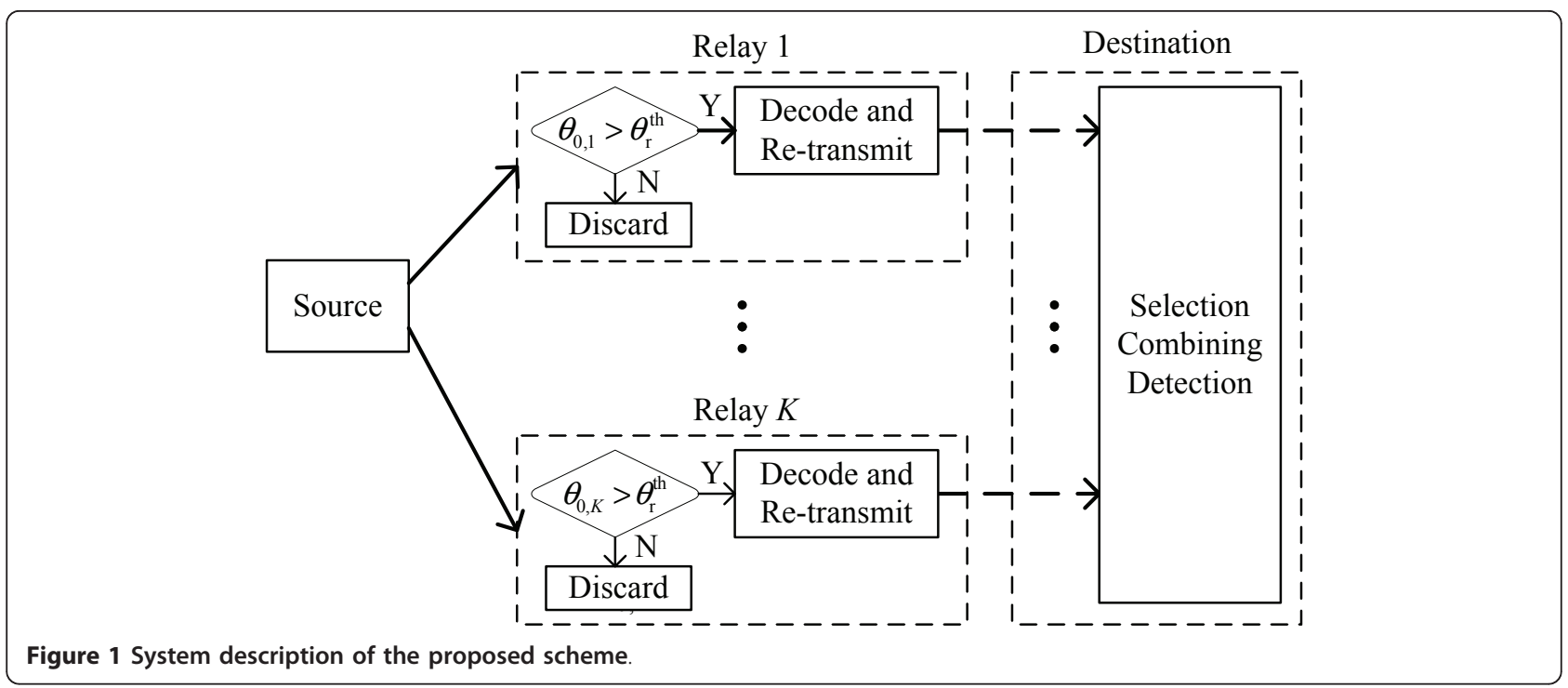

$$
\begin{aligned}
& y_{0, i, 0}=\left(1-x_{0}\right) \sqrt{E_{0}} h_{0, i}+n_{0, i, 0}, \\
& y_{0, i, 1}=x_{0} \sqrt{E_{0}} h_{0, i}+n_{0, i, 1},
\end{aligned}
$$

where $h_{0, i}$ and $n_{0, i, k}$ denote the channel fading coefficient between node 0 and node $i$ and the noise component at node $i$, respectively. $E_{0}$ is the average transmitted symbol energy of the source. The third subscript $k \in\{0$, $1\}$ in (1) and (2) denotes the two frequency subbands used in BFSK signaling. Furthermore, the source symbol $x_{0}=0$ if the first frequency subband is used and $x_{0}=1$ if the second frequency subband is used.

With noncoherent BFSK, signal detection at the $i$ th relay node is carried out by simply comparing the signal energies received in the two subbands. As such the instantaneous magnitude of the energy difference in the two subbands, namely $\theta_{0, i}=\left.|| y_{0, i, 0}\right|^{2}-\left|y_{0, i, 1}\right|^{2} \mid$, serves as a reliability measure of the detection at the $i$ th relay. Similar to [26], node $i$ only decodes and retransmits a BFSK signal if $\theta_{0, i}>\theta_{\mathrm{r}}^{\text {th }}$, where $\theta_{\mathrm{r}}^{\text {th }}$ is some fixed threshold value to be determined. If node $i$ transmits in the second phase, the received signals at the destination in the two subbands are given by

$$
\begin{aligned}
& y_{i, K+1,0}=\left(1-x_{i}\right) \sqrt{E_{i}} h_{i, K+1}+n_{i, K+1,0}, \\
& y_{i, K+1,1}=x_{i} \sqrt{E_{i}} h_{i, K+1}+n_{i, K+1,1},
\end{aligned}
$$

where $E_{i}$ is the average transmitted symbol energy sent by node $i$ and $n_{i, K+1, k}$ is the noise component at the destination in the second phase. Note that if the $i$ th relay makes a correct detection, then $x_{i}=x_{0}$. Otherwise $x_{i} \neq x_{0}$. On the other hand, when $\theta_{0, i}<\theta_{\mathrm{r}}^{\text {th }}$, node $i$ remains silent. In this case, the outputs in the two subbands are given by

$$
\begin{aligned}
& y_{i, K+1,0}=n_{i, K+1,0}, \\
& y_{i, K+1,1}=n_{i, K+1,1} .
\end{aligned}
$$

After receiving all the signals from the relays, the destination chooses only one signal with the largest magnitude of the energy difference in the two subbands to decode. In other words, the signal from node $i$ is chosen if $\max _{j \neq i} \theta_{j, K+1}<\theta_{i, K+1}$ where $\theta_{j, K+1}=\left.|| y_{j, K+1,0}\right|^{2}-\mid y_{j, K}$ $+1,\left.1\right|^{2} \mid, j=1, \ldots, K$. The detector is of the form:

$$
\Lambda=\left|y_{i, K+1,0}\right|^{2}-\left|y_{i, K+1,1}\right|^{2} \stackrel{0}{\gtrless} 0 .
$$

The next section derives the average BER for a general network, i.e., a network with arbitrary qualities of source-relay and relay-destination links. Using the derived BER, the optimum thresholds can then be numerically found.

\section{BER analysis and optimization of threshold and power allocation}

Let the noise components at the relays and destination be modeled as ${ }^{\mathrm{b}}$ i.i.d. $C \mathcal{N}\left(0, N_{0}\right)$ random variables. The channel between any two nodes is Rayleigh flat fading, modeled as $C \mathcal{N}\left(0, \sigma_{i, j}^{2}\right)$, where $i, j$ refer to transmit and receive nodes, respectively. The instantaneous received SNR for the transmission from node $i$ to node $j$ is given as $\gamma_{i, j}=E_{i}\left|h_{i, j}\right|^{2} / N_{0}$ and the corresponding average SNR is $\bar{\gamma}_{i, j}=E_{i} \sigma_{i, j}^{2} / N_{0}$. With Rayleigh fading, the pdf of $\gamma_{i, j}$ is $f_{i, j}\left(\gamma_{i, j}\right)=\frac{1}{\bar{\gamma}_{i, j}} e^{-\gamma i, j} \bar{\gamma}_{i, j}$. 
Recall that the destination selects only one signal among $K$ received signals to decode. The selected relay might forward a correct bit, an incorrect bit or remain silent in the second phase. Therefore, there are three different cases that result in different BERs at the destination. We parameterize the three cases by $\Theta \in\{1,2,3\}$ where $\Theta=1, \Theta=2$, and $\Theta=3$ are the events that the selected relay forwards a correct bit, an incorrect bit and remains silent, respectively. By using the law of total probability, the average BER with a given threshold $\theta_{\mathrm{r}}^{\text {th }}$ can be expressed as

$$
\operatorname{BER}\left(\theta_{\mathrm{r}}^{\text {th }}\right)=\sum_{i=1}^{3} P(\varepsilon, \Theta=i)
$$

where $P(\varepsilon, \Theta=i)$ is the average BER at the destination in the case $\Theta=i$.

To compute all the terms in (8), divide the set $S_{\text {relay }}=$ $\{1,2, \ldots, K\}$ of $K$ relays into three disjoint subsets $\Omega_{1}, \Omega_{2}$, and $\Omega_{3}$, which include the relays that forward a correct bit, an incorrect bit, and remain silent in the second phase, respectively. Clearly, $K=\left|\Omega_{1}\right|+\left|\Omega_{2}\right|+\left|\Omega_{3}\right|$ where $|\Omega|$ denotes the cardinality of set $\Omega$. Without loss of generality, assume that the transmitted information bit is "0". Also let $W_{m}\left(m \in \Omega_{1}\right), V_{n}\left(n \in \Omega_{2}\right)$ and $R_{l}\left(l \in \Omega_{3}\right)$ denote the energy differences in the two subbands measured at the destination for relay-destination links involving the relays in sets $\Omega_{1}, \Omega_{2}$ and $\Omega_{3}$, respectively. Obviously $P(\varepsilon, \Theta=i)$ can be calculated as follows:

$$
P(\varepsilon, \Theta=i)=\sum_{\Omega_{1} \in \mathcal{P}\left(S_{\text {reday }}\right)} \sum_{\Omega_{2} \in \mathcal{P}\left(S_{\text {reday }}\right)\left(\Omega_{1}\right)} P_{\Omega_{1}, \Omega_{2}, \Omega_{3}}(\varepsilon, \Theta=i) P\left(\Omega_{1}, \Omega_{2}, \Omega_{3}\right) .
$$

where $P_{\Omega_{1}, \Omega_{2}, \Omega_{3}}(\varepsilon, \Theta=i)$ and $P\left(\Omega_{1}, \Omega_{2}, \Omega_{3}\right)$ denote the conditional BER and case probability for the specific set $\left(\Omega_{1}, \Omega_{2}, \Omega_{3}\right)$. The notation $\mathcal{P}(A)$ means the power set of its argument, i.e., the set of all its subsets (including the empty set $\varnothing$ ). $A \backslash B$ denotes the relative complement of the set $B$ in the set $A$.

First, according to Lemmas 2 and 4 in [26], the probability density functions (pdfs) of $W_{m}, V_{n}$ and $R_{l}$ are given, respectively, by

$$
\begin{aligned}
& f_{W_{m}}(x)= \begin{cases}\frac{1}{2+\bar{\gamma}_{m, K+1}} \mathrm{e}^{-x /\left(1+\bar{\gamma}_{m, K+1}\right)}, & x \geq 0 \\
\frac{1}{2+\bar{\gamma}_{m, K+1}} \mathrm{e}^{x}, & x<0\end{cases} \\
& f_{V_{n}}(x)= \begin{cases}\frac{1}{2+\bar{\gamma}_{n, K+1}} \mathrm{e}^{-x}, & x \geq 0 \\
\frac{1}{2+\bar{\gamma}_{n, K+1}} \mathrm{e}^{x /\left(1+\bar{\gamma}_{2}\right)}, & x<0\end{cases} \\
& f_{R_{l}}(x)= \begin{cases}\frac{1}{2} \mathrm{e}^{-x}, & x \geq 0 \\
\frac{1}{2} \mathrm{e}^{x}, & x<0\end{cases}
\end{aligned}
$$

It then follows that

$$
\begin{aligned}
& f_{\left|W_{m}\right|}(x)=\frac{1}{2+\bar{\gamma}_{m, K+1}}\left(\mathrm{e}^{-x /\left(1+\bar{\gamma}_{m, K+1}\right)}+\mathrm{e}^{-x}\right), x \geq 0 \\
& f_{\left|V_{n}\right|}(x)=\frac{1}{2+\bar{\gamma}_{n, K+1}}\left(\mathrm{e}^{-x /\left(1+\bar{\gamma}_{n, K+1}\right)}+\mathrm{e}^{-x}\right), x \geq 0 \\
& f_{\left|R_{l}\right|}(x)=\mathrm{e}^{-x}, x \geq 0
\end{aligned}
$$

\subsection{Case probability}

The probability of occurrence for the specific set $\left\{\Omega_{1}\right.$, $\Omega_{2}, \Omega_{3}$ \} can be determined to be

$$
\begin{aligned}
P\left(\Omega_{1}, \Omega_{2}, \Omega_{3}\right)= & \prod_{i \in\left(\Omega_{1} \cup \Omega_{2}\right)}\left[1-I_{1}\left(\theta_{\mathrm{r}}^{\text {th }}, \bar{\gamma}_{0, i}\right)\right] \prod_{i \in \Omega_{1}}\left[1-I_{2}\left(\theta_{\mathrm{r}}^{\text {th }}, \bar{\gamma}_{0, i}\right)\right] \\
& \times \prod_{i \in \Omega_{2}} I_{2}\left(\theta_{\mathrm{r}}^{\text {th }}, \bar{\gamma}_{0, i}\right) \prod_{i \in \Omega_{3}} I_{1}\left(\theta_{\mathrm{r}}^{\text {th }}, \bar{\gamma}_{0, i}\right)
\end{aligned}
$$

where $A \cup B$ denotes the union of sets $A$ and $B$. The function $I_{1}\left(\theta_{\mathrm{r}}^{\text {th }}, \bar{\gamma}_{0, i}\right)$ is the probability that the magnitude of the energy difference in the two subbands at node $i$ is smaller than the threshold, i.e., $\theta_{0, i}<\theta_{\mathrm{r}}^{\text {th }}$. The pdf of $\theta_{0, i}$ is given in Lemma 2 of [26], which is used to obtain the following expression for $I_{1}\left(\theta_{\mathrm{r}}^{\text {th }}, \bar{\gamma}_{0, i}\right)$ :

$$
\begin{aligned}
I_{1}\left(\theta_{\mathrm{r}}^{\text {th }}, \bar{\gamma}_{0, i}\right) & =\int_{0}^{\theta_{t}^{\mathrm{th}}} f_{\theta_{0, i}}(x) \mathrm{d} x=\int_{0}^{\theta_{\mathrm{r}}^{\mathrm{h}}} \frac{1}{2+\bar{\gamma}_{0, i}}\left(\mathrm{e}^{-x /\left(1+\bar{\gamma}_{0, i}\right)}+\mathrm{e}^{-x}\right) \mathrm{d} x \\
& =\frac{1+\bar{\gamma}_{0, i}}{2+\bar{\gamma}_{0, i}}\left[1-\mathrm{e}^{-\theta_{\mathrm{r}}^{\mathrm{th}} /\left(1+\bar{\gamma}_{0, i}\right)}\right]+\frac{1}{2+\bar{\gamma}_{0, i}}\left[1-\mathrm{e}^{-\theta_{\mathrm{r}}^{\mathrm{th}}}\right]
\end{aligned}
$$

On the other hand, $I_{2}\left(\theta_{\mathrm{r}}^{\text {th }}, \bar{\gamma}_{0, i}\right)$ is the probability of error at node $i, i=1, \ldots, K$, given that the magnitude of the energy difference in the two subbands is larger than the threshold, i.e., $\theta_{0, i}>\theta_{\mathrm{r}}^{\text {th }}$. Therefore, $I_{2}\left(\theta_{\mathrm{r}}^{\text {th }}, \bar{\gamma}_{0, i}\right)$ can be computed as

$$
I_{2}\left(\theta_{\mathrm{r}}^{\mathrm{th}}, \bar{\gamma}_{0, i}\right)=\frac{1}{1-I_{1}\left(\theta_{\mathrm{r}}^{\mathrm{th}}, \bar{\gamma}_{0, i}\right)} \int_{-\infty}^{-\theta^{\mathrm{an}}} \frac{1}{2+\bar{\gamma}_{0, i}} \mathrm{e}^{-x} \mathrm{~d} x=\frac{1}{2+\bar{\gamma}_{0, i}} \frac{1}{1-I_{1}\left(\theta_{\mathrm{r}}^{\mathrm{th}}, \bar{\gamma}_{0, i}\right)} \mathrm{e}^{-\theta_{\mathrm{t}}^{\mathrm{th}}}
$$

\subsection{Case $\Theta=1$}

Next we compute the average BER for $\Theta=1$ conditioned on $\left\{\Omega_{1}, \Omega_{2}, \Omega_{3}\right\}$. In this case, the selected relay forwards a correct bit. This means that an error occurs at the destination if among the $K$ statistics $W_{m}, V_{n}$ and $R_{l}$, the one with the largest magnitude is one of $W_{m}$ and negative. Thus, the conditional BER can be written as

$$
\begin{aligned}
P_{\Omega_{1}, \Omega_{2}, \Omega_{3}}(\varepsilon, \Theta=1) & =\sum_{m \in \Omega_{1}} P\left(\max _{i \neq m}\left(\left|W_{i}\right|,\left|V_{n}\right|,\left|R_{l}\right|\right)<\left|W_{m}\right|, W_{m}<0\right) \\
& =\sum_{m \in \Omega_{1}} P\left(\max _{i \neq m}\left(\left|W_{i}\right|,\left|V_{n}\right|,\left|R_{l}\right|\right)+W_{m}<0\right) \\
& =\sum_{m \in \Omega_{1}} P\left(\bar{W}_{m}+W_{m}<0\right)
\end{aligned}
$$


where $\bar{W}_{m}=\max _{i \neq m}\left(\left|W_{i}\right|,\left|V_{n}\right|,\left|R_{l}\right|\right)$. The pdf of $\bar{W}_{m}$ can be found as follows:

$$
\begin{aligned}
& f_{\bar{W}_{m}}(x)=\frac{\mathrm{d}}{\mathrm{d} x} P\left(\bar{W}_{m}<x\right)=\frac{\mathrm{d}}{\mathrm{d} x}\left(\prod_{i \in\left(\Omega_{1} \backslash\langle m\}\right)} F_{\left|W_{i}\right|}(x) \prod_{n \in \Omega_{2}} F_{\left|V_{n}\right|}(x) \prod_{l \in \Omega_{3}} F_{\left|R_{\mid}\right|}(x)\right) \\
& =\sum_{i \in\left(\left(\Omega_{1} \cup \Omega_{2}\right) \backslash\langle m\}\right)} f_{\left|W_{i}\right|}(x) \prod_{j \in\left(\left(\Omega_{1} \cup \Omega_{2}\right) \backslash\langle m, i\}\right)} F_{\left|W_{j}\right|}(x) \prod_{l \in \Omega_{3}} F_{\left|R_{l}\right|}(x) \\
& +\sum_{i \in \Omega_{3}} f_{\left|R_{i}\right|}(x) \prod_{\left.l \in\left(\Omega_{3} \backslash \backslash i i\right\}\right)} F_{\left|R_{i}\right|}(x) \prod_{j \in\left(\left(\Omega_{1} \cup \Omega_{2}\right) \backslash\langle m\}\right)} F_{\left|W_{j}\right|}(x)
\end{aligned}
$$

It then follows that

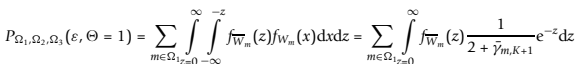

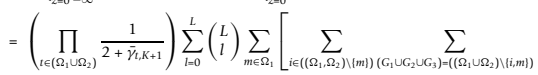

$$
\begin{aligned}
& \left((-1)^{\left|G_{2}\right|+\left|G_{3}\right|+\mid} \prod_{t \in G_{1}}\left(2+\bar{\gamma}_{t}, K_{1}\right) \prod_{t \in G_{2}}\left(1+\bar{\gamma}_{t, K+1}\right)\right) \\
& \left.\times\left(\frac{1}{\sum_{\in \in\left(G_{2}, i \mid i\right)} \frac{1}{1+\tilde{\gamma}_{L}, K+1}+\left|G_{3}\right|+l+1}+\frac{1}{\sum_{l \in G_{2}} \frac{1}{1+\tilde{\gamma}_{L}, K+1}+\left|G_{3}\right|+1+2}\right)\right] \\
& +\left(\prod_{t \in\left(\Omega_{1} \cup \Omega_{2}\right)} \frac{1}{2+\bar{\gamma}_{L}, K_{+1}}\right) \sum_{l=0}^{L-1}\left(\begin{array}{l}
L-1 \\
l
\end{array}\right) \sum_{m \in \Omega_{1}}\left[\sum_{i \in \Omega_{3}} \sum_{\left(G_{1} \cup G_{2} \cup G_{3}\right)=\left(\left(\Omega_{1} \cup \Omega_{2}\right) \backslash\langle m|\right)}\right. \\
& \left((-1)^{\left|\mathrm{G}_{2}\right|+\left|\mathrm{G}_{3}\right|+t} \prod_{t \in \mathrm{G}_{1}}\left(2+\bar{\gamma}_{t, K+1}\right) \prod_{t \in \mathrm{G}_{2}}\left(1+\bar{\gamma}_{t, K+1}\right)\right)
\end{aligned}
$$

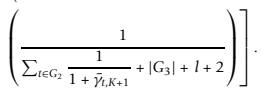

where $\left(G_{1} \cup G_{2} \cup G_{3}\right)=\Omega$ means that $G_{1}, G_{2}$ and $G_{3}$ are three disjoint subsets of $\mathcal{P}(\Omega)$ and the union of those disjoint subsets is $\Omega$.

\subsection{Case $\Omega=2$}

In this case, the selected relay forwards an incorrect bit, i.e., an error occurs if among the $K$ statistics $W_{m}, V_{n}$ and $R_{l}$, the one with the largest magnitude is one of $V_{n}$ and negative. Let $\bar{V}_{n}=\max _{i \neq n}\left(\left|W_{m}\right|,\left|V_{i}\right|,\left|R_{l}\right|\right)$. It can be shown that the pdf of $\bar{V}_{n}$ is as (20) by replacing $m$ by $n$. Similar to the case $\Theta=1$, one has

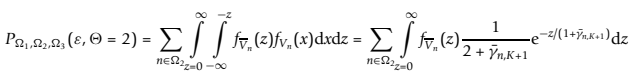

$$
\begin{aligned}
& =\left(\prod_{t \in\left(\Omega_{1} \cup \Omega_{2}\right)} \frac{1}{2+\bar{\gamma}_{1}, K+1}\right) \sum_{l=0}^{L}\left(\begin{array}{l}
L \\
l
\end{array}\right) \sum_{n \in \Omega_{2}}\left[\sum_{i \in\left(\left(\Omega_{1}, \Omega_{2}\right) \backslash \backslash(n)\right)} \sum_{\left(G_{1} \cup G_{2} \cup G_{3}\right)=\left(\left(\Omega_{1} \cup \Omega_{2}\right) \backslash\langle i, n|\right)}\right.
\end{aligned}
$$

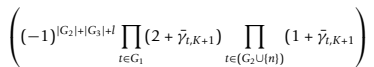

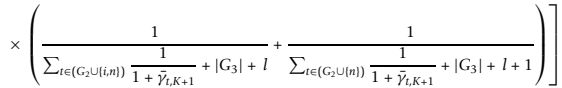

$$
\begin{aligned}
& +\left(\prod_{t \in\left(\Omega_{2} \cup \Omega_{2}\right)} \frac{1}{2+\tilde{\gamma}_{t, K+1}}\right) \sum_{l=0}^{L-1}\left(\begin{array}{c}
L-1 \\
l
\end{array}\right) \sum_{n \in \Omega_{2}}\left[\sum_{i \in \Omega_{\Omega}} \sum_{i\left(G_{1} \cup G_{2} \cup G_{3}\right)=\left(\left(\Omega_{1}, \Omega_{2}\right) \backslash \backslash(n)\right)}\right. \\
& \left.\left((-1)^{\left|G_{2}\right|+\left|G_{3}\right|+t} \prod_{t \in G_{1}}\left(2+\bar{\gamma}_{t, K+1}\right) \prod_{t \in\left(G_{2} \cup(n n)\right)}\left(1+\bar{\gamma}_{t, K+1}\right)\right)\left(\frac{1}{\sum_{t \in\left(G_{2} \cup(n) \mid\right)} \frac{1}{1+\bar{\gamma}_{t, K+1}}+\left|G_{3}\right|+l+1}\right)\right]
\end{aligned}
$$

\subsection{Case $\Theta=3$}

Different from cases $\Theta=1$ and $\Theta=2$, in this case, the selected relay remains silent in the second phase, i.e., it is one of the relays in $\Omega_{3}$. The conditional BER is

$$
\begin{aligned}
P_{\Omega_{1}, \Omega_{2}, \Omega_{3}}(\varepsilon, \Theta=3) & =\sum_{l \in \Omega_{3}} P\left(\max _{i \neq l}\left(\left|W_{m}\right|,\left|V_{n}\right|,\left|R_{i}\right|\right)<\left|R_{l}\right|, R_{l}<0\right) \\
& =\sum_{l \in \Omega_{3}} P\left(\max _{i \neq l}\left(\left|W_{m}\right|,\left|V_{n}\right|,\left|R_{i}\right|\right)+R_{l}<0\right) \\
& =\sum_{l \in \Omega_{3}} P\left(\bar{R}_{l}+R_{l}<0\right)
\end{aligned}
$$

where $\bar{R}_{l}=\max _{i \neq l}\left(\left|W_{m}\right|,\left|V_{n}\right|,\left|R_{i}\right|\right)$. The pdf of $\bar{R}_{l}$ can be found as follows:

$$
\begin{aligned}
& f_{\bar{R}_{l}}(x)=\frac{\mathrm{d}}{\mathrm{d} x} P\left(\bar{R}_{l}<x\right)=\frac{\mathrm{d}}{\mathrm{d} x}\left(\prod_{m \in \Omega_{1}} F_{\left|W_{m \mid}\right|}(x) \prod_{n \in \Omega_{2}} F_{\left|V_{V_{1}}\right|}(x) \prod_{i \in\left(\Omega_{3} \backslash[l]\right)} F_{\left|R_{i}\right|}(x)\right) \\
& =\sum_{m \in\left(\Omega_{1} \cup \Omega_{2}\right)} f_{W_{m} \mid}(x) \prod_{j \in\left(\left(\Omega_{1} \cup \Omega_{2}\right) \backslash(m)\right]} F_{i W_{j} \mid}(x) \prod_{i \in\left(\Omega_{3} \backslash(l l)\right)} F_{R_{i} \mid}(x) \\
& +\sum_{i \in \in\left(\Omega_{3} \backslash\langle l]\right)} f_{\mid R_{i}}(x) \prod_{j \in\left(\Omega_{3} \backslash\langle i, l]\right)} F_{\left|R_{j}\right|}(x) \prod_{m \in\left(\Omega_{1} \cup \Omega_{2}\right)} F_{\left|W_{m}\right|}(x)
\end{aligned}
$$

Therefore,

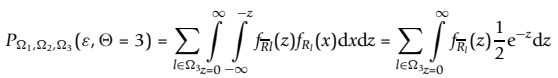

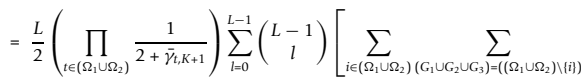

$$
\begin{aligned}
& \left((-1)^{\left|C_{2}+\right| C_{S} \mid t+} \prod_{t \in G_{1}}\left(2+\bar{\gamma}_{t, K+1}\right) \prod_{t \in C_{2}}\left(1+\bar{\gamma}_{t, K+1}\right)\right. \\
& \left.\left(\frac{1}{\sum_{\epsilon \in\left(C_{2} U(i)\right.} \frac{1}{1+\tilde{\gamma}_{4}, K+1}+\left|G_{3}\right|+l+1}+\frac{1}{\sum_{\ell \in G_{2}} \frac{1}{1+\tilde{y}_{1}, K+1}+\left|G_{3}\right|+l+2}\right)\right]
\end{aligned}
$$

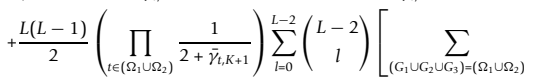

$$
\begin{aligned}
& \left((-1)^{\left|G_{2}\right|+\left|G_{3}\right|+t} \prod_{l \in G_{1}}\left(2+\bar{\gamma}_{t, K+1)} \prod_{l \in C_{2}}\left(1+\bar{\gamma}_{t, K+1}\right)\right)\left(\frac{1}{\sum_{i \in C_{2}} \frac{1}{1+\tilde{\gamma}_{t, K+1}}+\left|G_{3}\right|+l+2}\right)\right]
\end{aligned}
$$

To summarize, all the expressions involved in the final expression of the average BER in (8) can be calculated analytically. Although final expression is quite involved and presents limited insights, it is simple enough to use in optimizing the threshold $\theta_{\mathrm{r}}^{\text {th }}$ to minimize the average BER of the network.

First, for a fixed power allocation among the source and relays, the optimization of the threshold value can be set up as follows:

$$
\hat{\theta}_{\mathrm{r}}^{\text {th }}=\arg \min _{\theta_{\mathrm{r}}^{\text {th }}} \operatorname{BER}\left(\theta_{\mathrm{r}}^{\text {th }}\right) .
$$

On the other hand, the total transmitted power of the network can also be optimally allocated to the source and relays. To this end, let the total signal energies at the source and relays be $E_{\text {total }}$ and the maximum signal energy that can be allocated to node $i$ as $E_{i, \max }$. Then, the joint optimization of the threshold $\theta_{\mathrm{r}}^{\text {th }}$ and power to minimize the average BER are as follows:

$$
\begin{aligned}
& \left(\hat{\theta}_{\mathrm{r}}^{\mathrm{th}}, \hat{E}_{0}, \hat{E}_{1}, \ldots, \hat{E}_{K}\right)=\arg \min _{\left(\theta_{\mathrm{r}}^{\mathrm{m},}, E_{0}, E_{1}, \ldots, E_{K}\right)} \operatorname{BER}\left(\theta_{\mathrm{r}}^{\mathrm{th}}, E_{0}, E_{1}, \ldots, E_{K}\right), \\
& \text { subject to }\left\{\begin{array}{l}
0 \leq E_{i} \leq E_{i, \max }, i=0, \ldots, K \\
\sum_{i=0}^{K} E_{i}=E_{\text {tota } 1}
\end{array}\right.
\end{aligned}
$$


With the closed-form expression of the average BER, the above optimization problems can be solved by optimization techniques such as the Lagrange method [27]. Unfortunately, the exponential terms in the final expressions render a closed-from solution intractable. The optimization problems in (26) and (27) are simply solved with the MATLAB Optimization Toolbox. ${ }^{\mathrm{C}}$ It should be pointed out that, without proving the BER function is convex, the solutions obtained by MATLAB might only locally optimum solutions. Nevertheless, plotting the BER function versus the threshold value for various power allocations shows that the objective function is convex. This strongly suggests that the solutions are globally optimum. Moreover, since the average BER formulated in (8) only requires information on the average SNRs of the source-relay and relay-destination links, the optimization problems can be solved off-line for typical sets of average SNRs and the obtained optimal threshold and/or power ratio values are stored in a look-up table.

\section{Simulation results}

In all the simulations the noise components at the relays and destination are modeled as i.i.d.

$\mathcal{C N}(0,1)$ random variables. For convenience, define $\boldsymbol{\sigma}^{2}=\left[\begin{array}{llll}\sigma_{0,1}^{2} & \ldots & \sigma_{0, K}^{2} \sigma_{1, K+1}^{2} \ldots \sigma_{K, K+1}^{2}\end{array}\right]$. Figure 2 plots the average BERs at the destination for different channel conditions and different number of relays. Here the threshold is simply chosen as $\theta_{\mathrm{r}}^{\text {th }}=2$ to verify that our BER analysis is valid for any threshold value. The transmitted powers are set to be the same for the source and relays. The figure shows that the analytical (shown in lines) and simulation (shown as marker symbols) results are identical, hence verifying our analysis in Section 3.

Next, Figure 3 compares the performance of the proposed scheme with that of PL scheme and the scheme in [26] in a two-relay network. The channel variances of all the transmission links in the network are set to be $\sigma^{2}=\left[\begin{array}{llll}1.5 & 1.5 & 1.5 & 1.5\end{array}\right]$. The node energy constraints are $E_{0, \max }=0.6 E_{\text {total }}, E_{i, \max }=0.3 E_{\text {total }}, i=1,2,3$. The figure shows that our proposed scheme with selection combining outperforms the PL scheme. This is expected since the continuous retransmission of relays in the PL scheme causes error propagation and hence limits its BER performance. Furthermore, it can also be seen that the relaying scheme proposed in this paper performs the same as the scheme in [26] under both cases of fixed and optimal power allocations. This is not a surprising observation either as it can be verified that in a tworelay network, whether selecting the best received signal or combining two received signals does not affect the decision at the destination. ${ }^{\mathrm{d}}$
Figure 4 shows the average BERs obtained by simulation for three different schemes in a three-relay cooperative network. ${ }^{\mathrm{e}}$ Here $\sigma^{2}=\left[\begin{array}{llllll}0.5 & 1.0 & 2.0 & 1.0 & 1.5 & 2.0\end{array}\right]$. From the figure, both the optimal threshold scheme and jointly optimal threshold and power-allocation scheme achieve better BER performances compared to the PL scheme. The percentages of total power spent for node $0,1,2$, and 3 are 52.47, 12.61, 15.59, and 19.33\%, respectively when the average power per node is $20 \mathrm{~dB}$. This optimum power allocation is reasonable intuitively satisfying since what it does is to allocate a big portion of the power to the source to reduce decoding errors at the relays. Then, more reliable relays are accordingly allocated more powers since the destination is expected to select the signal from the relay that forwards a correct bit. Similar results are observed for other values of the total power.

Figure 5 presents performance improvement of the proposed scheme in a five-relay network when the variances of Rayleigh fading channels are set to be $\sigma^{2}=$ $\left[\begin{array}{llllllllll}3.5 & 2.5 & 0.1 & 1.5 & 0.4 & 3.5 & 2.5 & 0.1 & 1.5 & 0.4\end{array}\right]$. The node energy constraints are set to be $E_{0, \max }=0.6 E_{\text {total }}, E_{i, \max }=$ $0.3 E_{\text {total }}, i=1, \ldots, 5$. An SNR gain of about $3 \mathrm{~dB}$ is observed at the BER level of $10^{-6}$ by the proposed scheme with the optimal threshold value when compared to the PL scheme. The figure also shows that jointly optimizing the threshold and power-allocation scheme can be further beneficial in the proposed network. Specifically a further gain of $2 \mathrm{~dB}$ can be realized when compared to the case of solely optimizing the threshold value. The results presented in Figure 5 are also intuitively satisfying. Since the relays are geographically distributed, the PL scheme suffers from more decision errors made at the relays that are far from the source. Setting a proper threshold at the relays and/or re-allocating the power between the source and the relays is therefore beneficial in this situation.

It should be pointed out that the proposed scheme can actually save some power compared to the PL scheme (similar to the scheme with two thresholds proposed in [26]). This has not been incorporated in the BER plots in Figures 3, 4 and 5, where the BER curves are plotted versus the average power assigned per node, rather than the average power consumed per node. Such a power saving is a direct consequence of the fact that a relay might be silent in the second phase. However, numerical results indicate that the power saving is significant only at low/medium SNR and without powerallocation optimization. ${ }^{\mathrm{f}}$ This is expected since a relay likely makes more errors at low/medium SNR and therefore remains silent in the second phase. On the other hand, with the joint optimization of the threshold and power ratio, more power will be allocated to the 


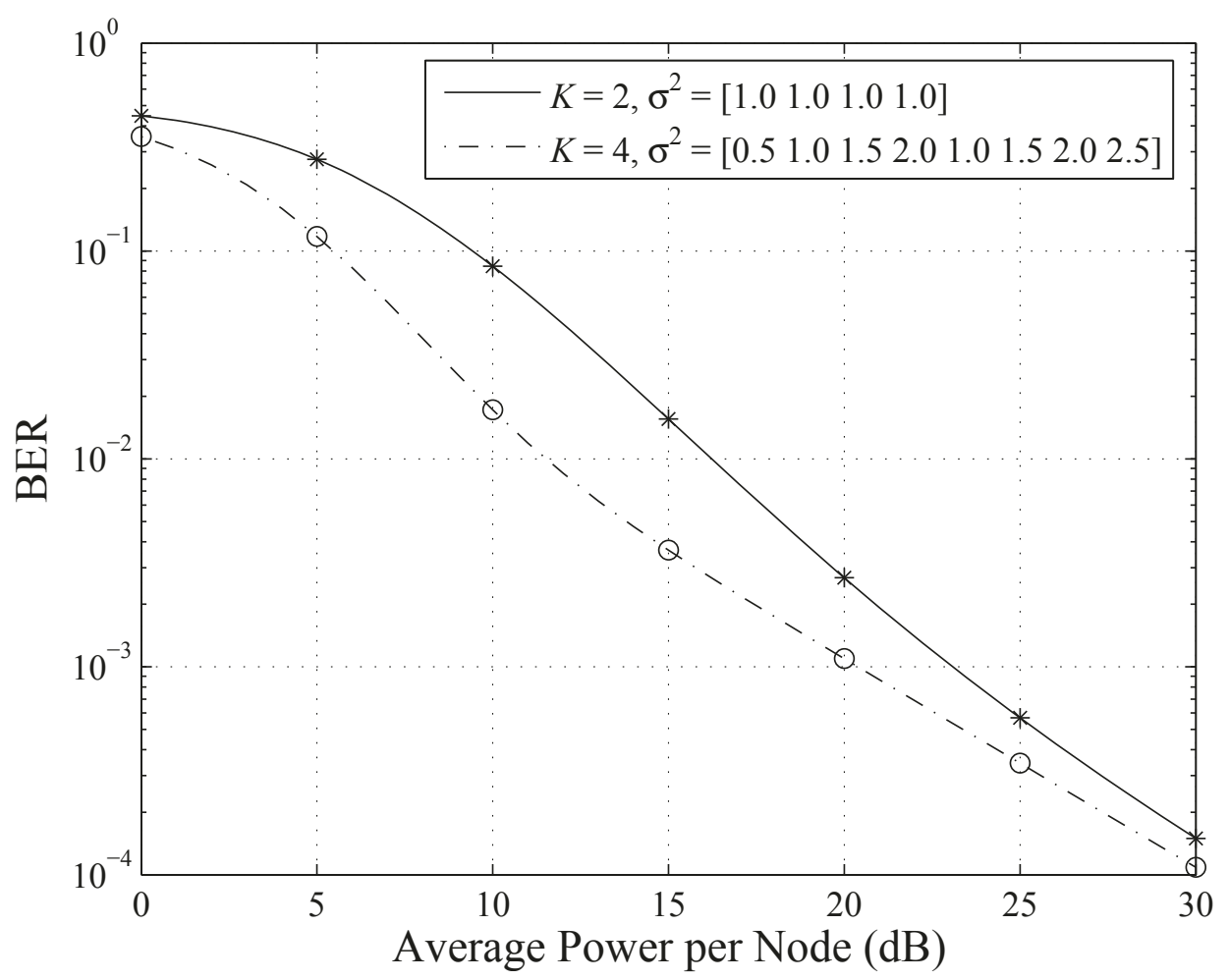

Figure 2 BERs of multiple-relay cooperative networks. Exact analytical values are shown in lines and simulation results are shown as marker symbols.

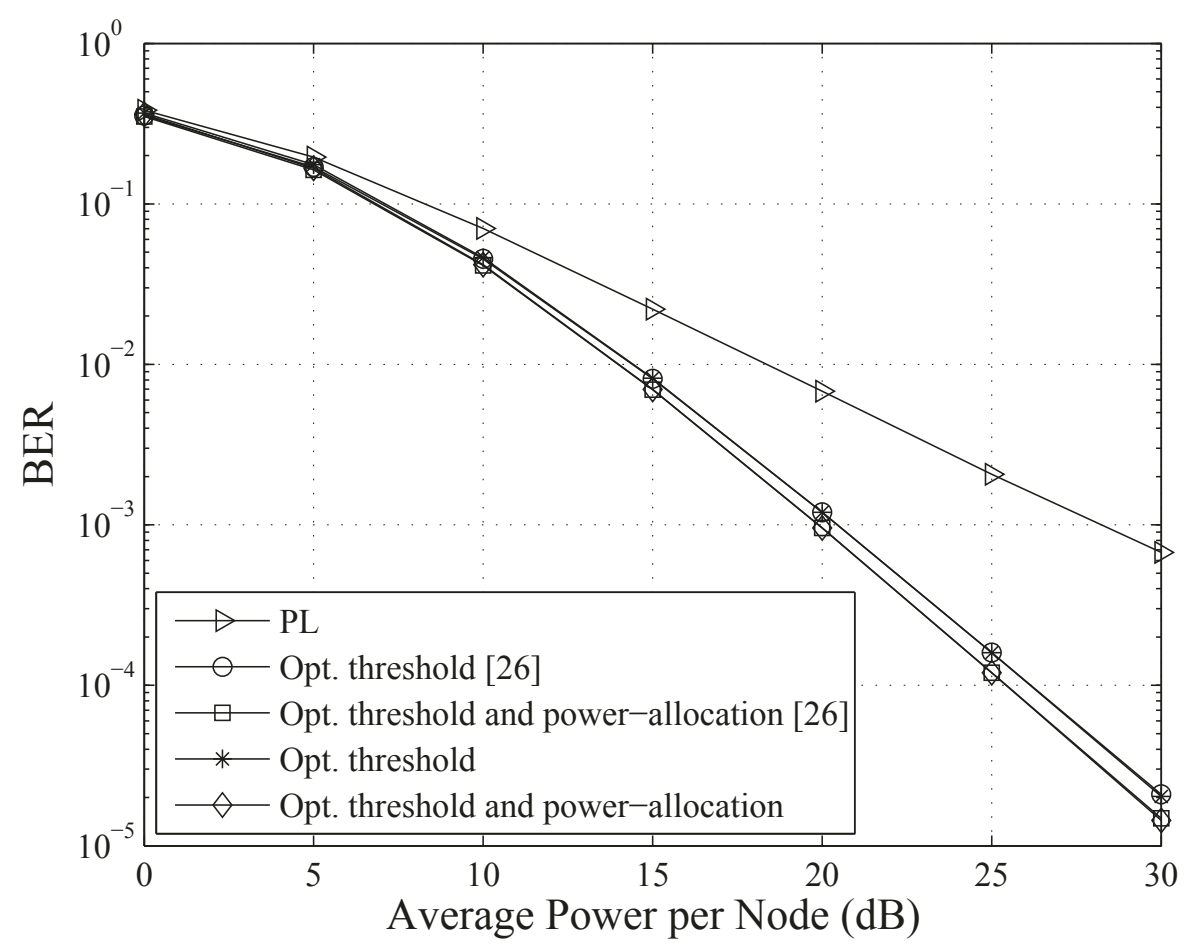

Figure 3 BERs of a two-relay network with different schemes when $\sigma^{2}=\left[\begin{array}{llll}1.5 & 1.5 & 1.5 & 1.5\end{array}\right]$ 


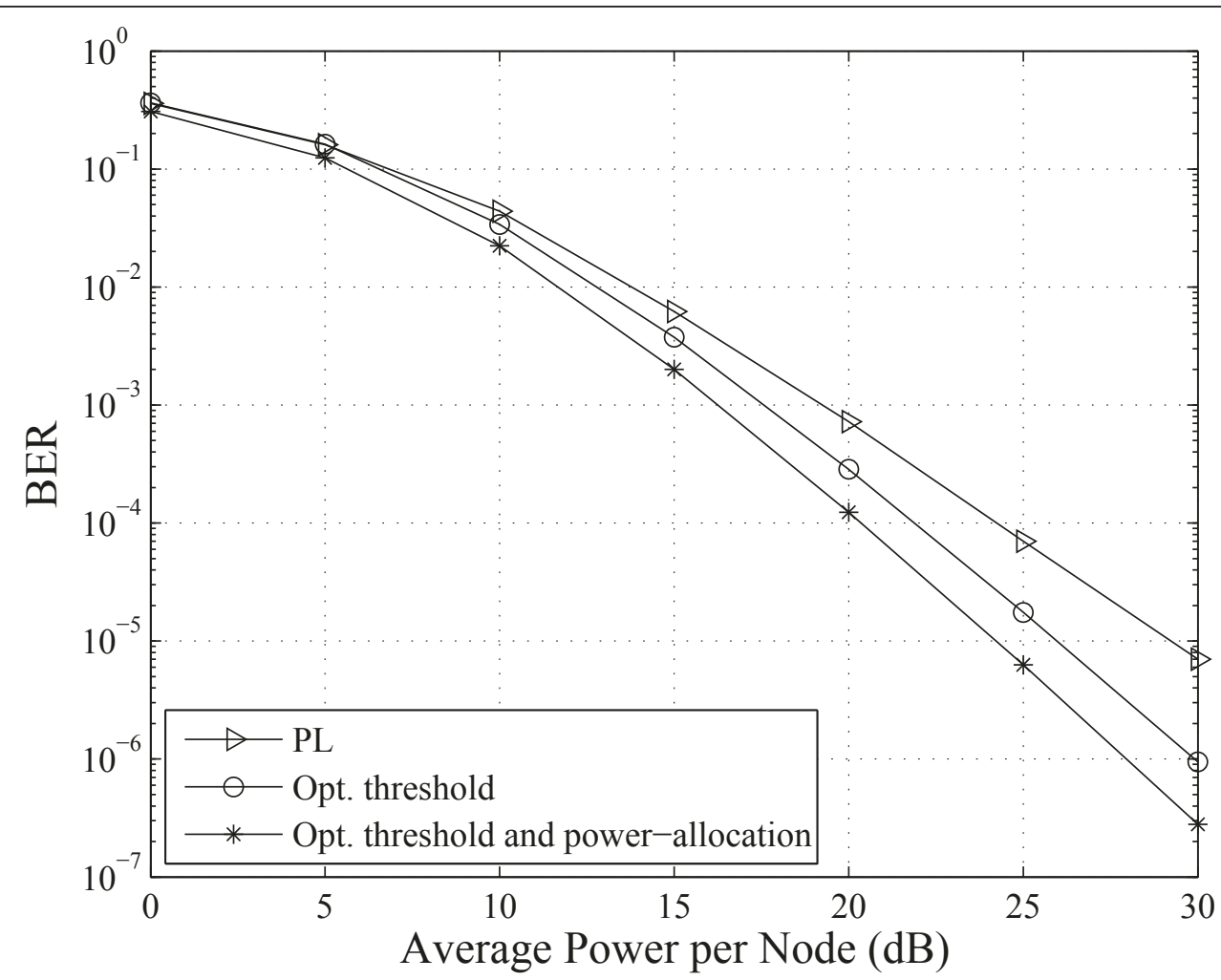

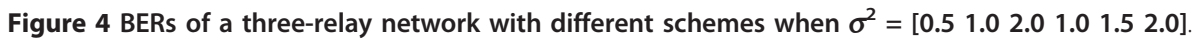

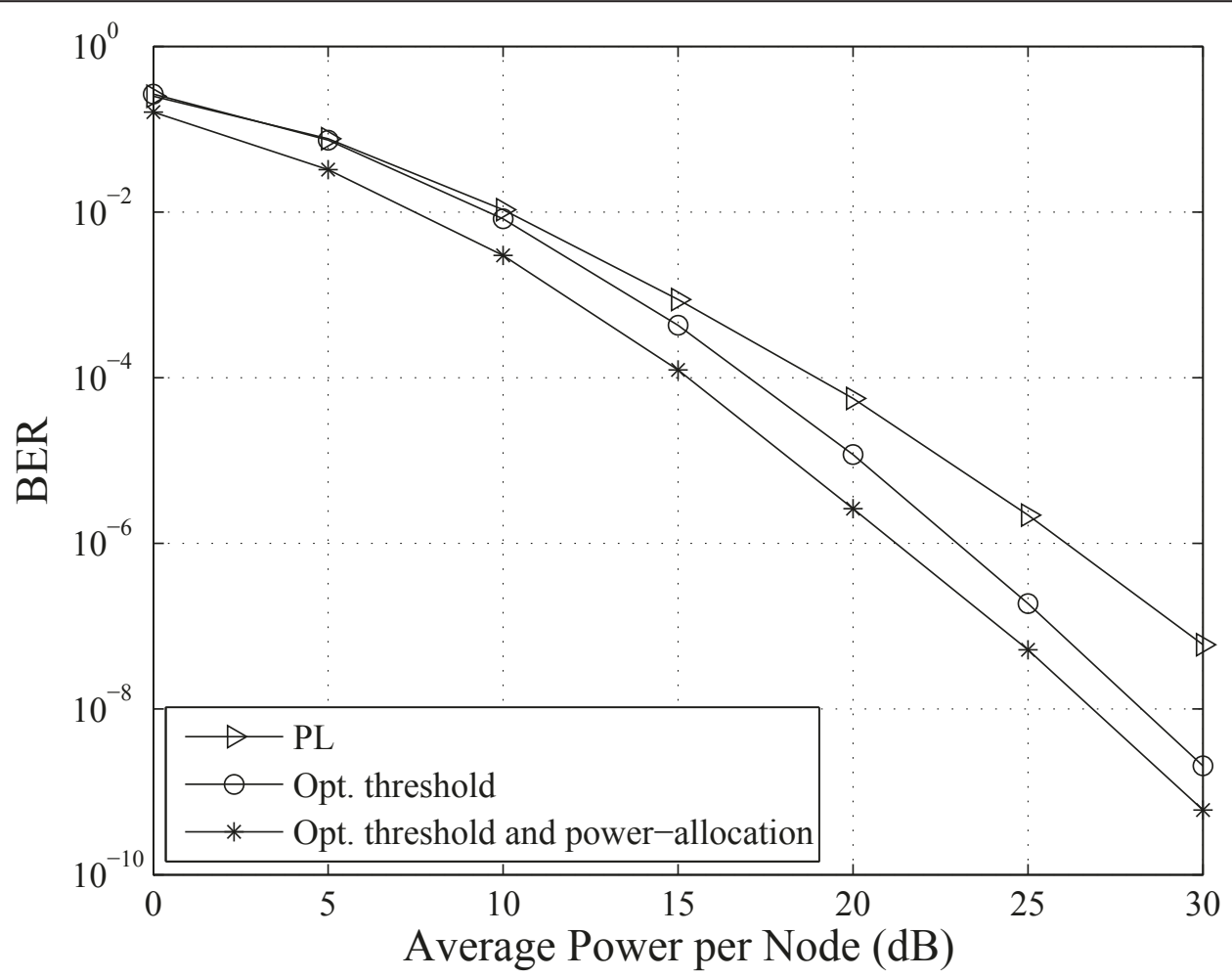

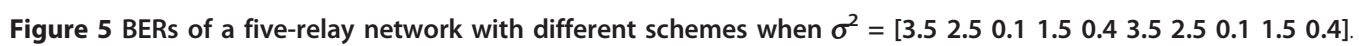


source to reduce decoding error at the relays, and hence the relays are more likely to retransmit in the second phase.

Finally, it should be mentioned that, in general, the diversity order of the network depends on the chosen threshold value. Unfortunately, a theoretical analysis of the diversity order is not available. Nevertheless, the obtained BER expression is simple enough to plot and one can examine the diversity order by observing the BER curve. In fact, examining the BER curves indicates that the proposed scheme (with optimal threshold/ power allocation) achieves the full diversity order.

\section{Conclusion}

In this paper, we have obtained the average BER expression for data transmission over a noncoherent cooperative network with $K+2$ nodes. BFSK is employed at both the source and relays to facilitate noncoherent communications. A single threshold is employed to select retransmitting relays. A relay retransmits the decoded signal to the destination if its decision variable is larger than a threshold. Otherwise, it remains silent. The destination chooses the received signal with the largest decision variable to decode the transmitted information (i.e., selection combining). With the obtained closed-form BER expression, the optimal threshold or jointly optimal threshold and power allocation are chosen to minimize the average BER. Simulation results were presented to corroborate the analysis. Performance comparison reveals that the proposed scheme outperforms the conventional scheme with a similar complexity.

\section{Endnotes}

${ }^{a}$ Considering orthogonal channels implies that one needs to trade multiplexing gain for error performance. ${ }^{\mathrm{b}} \mathcal{C N}\left(0, \sigma^{2}\right)$ denotes a circularly symmetric complex Gaussian random variable with variance $\sigma^{2}$. 'Specifically, we made use of the routine "fmincon", which is designed to find the minimum of a given constrained nonlinear multivariable function. ${ }^{\mathrm{d}}$ Without loss of generality, assume that the first branch is selected to decode the transmitted information, i.e., $\theta_{1,3}>\theta_{2,3}$. The decision is of the form: $\Lambda_{\mathrm{SC}}=\left|y_{1,3,0}\right|^{2}-\left|y_{1,3,1}\right|^{2} \stackrel{0}{\gtrless} 0$. With the scheme in [26], the decision is as $\Lambda_{[26]}=\left|y_{1,3,0}\right|^{2}-\left|y_{1,3,1}\right|^{2}+\left|y_{2,3,0}\right|^{2}-\left|y_{2,3,1}\right|^{2} \underset{1}{\gtrless} 0$. One can easily verify that both decisions give the same result as follows: $\theta_{1,3}>\theta_{2,3} \Leftrightarrow\left(\left|y_{1,3,0}\right|^{2}-\left|y_{1,3,1}\right|^{2}+\left|y_{2,3,0}\right|^{2}-\mid\right.$ $\left.\left.y_{2,3,1}\right|^{2}\right)\left(\left|y_{1,3,0}\right|^{2}-\left|y_{1,3,1}\right|^{2}-\left|y_{2,3,0}\right|^{2}+\left|y_{2,3,1}\right|^{2}\right)>0 \Leftrightarrow \Lambda_{[26]}$ $\left(2 \Lambda_{\mathrm{SC}}-\Lambda_{[26]}\right)>0$. It means that if $\Lambda_{[26]}>0$, then $\Lambda_{\mathrm{SC}}$ $>0$. Otherwise, if $\Lambda_{[26]}<0$, then $\Lambda_{\mathrm{SC}}<0$. ${ }^{e} \mathrm{We}$ are aware that the comparison between the PL scheme and jointly optimal threshold and power-allocation scheme might be unfair. Since reference [25] does not provide an average BER expression in a cooperative network with more than one relay, it is not possible to systematically obtain the optimal power allocation for the PL scheme. However, we believe that our proposed scheme has a better BER performance than the PL scheme with/without

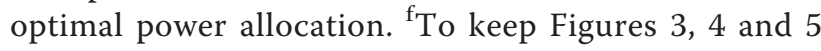
readable the BER curves taking into account power saving are not included.

\section{Acknowledgements}

This work was supported by an NSERC Discovery Grant.

\section{Authors' contributions}

HX proposed the new relaying protocol, carried out the simulations and participated in the draft of the manuscript. HH supervised the research and revised the manuscript. All authors read and approved the final manuscript.

\section{Competing interests}

The authors declare that they have no competing interests.

Received: 22 February 2011 Accepted: 21 September 2011

Published: 21 September 2011

\section{References}

1. J Laneman, G Wornell, Distributed space-time-coded protocols for exploiting cooperative diversity in wireless networks. IEEE Trans Inform Theory. 49, 2415-2425 (2003). doi:10.1109/TIT.2003.817829

2. A Sendonaris, E Erkip, B Aazhang, User cooperation diversity, Part I: system description. IEEE Trans Commun. 51(11), 1927-1938 (2003). doi:10.1109/ TCOMM.2003.818096

3. A Sendonaris, E Erkip, B Aazhang, User cooperation diversity, Part II: Implementation aspects and performance analysis. IEEE Trans Commun. 51(11), 1939-1948 (2003). doi:10.1109/TCOMM.2003.819238

4. A Bletsas, A Khisti, D Reed, A Lippman, A simple cooperative diversity method based on network path selection. IEEE J Sel Areas Commun. 24, 659-672 (2006)

5. D Michalopoulos, G Karagiannidis, Performance analysis of single relay selection in Rayleigh fading. IEEE Trans Wirel Commun. 7, 3718-3724 (2008)

6. F Onat, A Adinoyi, Y Fan, H Yanikomeroglu, J Thompson, I Marsland, Threshold selection for SNR-based selective digital relaying in cooperative wireless networks. IEEE Trans Wirel Commun. 7, 4226-4237 (2008)

7. F Onat, Y Fan, H Yanikomeroglu, J Thompson, Asymptotic BER analysis of threshold digital relaying schemes in cooperative wireless systems. IEEE Trans Wirel Commun. 7, 4938-4947 (2008)

8. J Laneman, D Tse, G Wornell, Cooperative diversity in wireless networks: efficient protocols and outage behavior. IEEE Trans Inf Theory. 50, 3062-3080 (2004). doi:10.1109/TIT.2004.838089

9. A Bletsas, H Shin, M Win, Cooperative communications with outage-optimal opportunistic relaying. IEEE Trans Wirel Commun. 6, 3450-3460 (2007)

10. MK Simon, M-S Alouini, Digital Communication Over Fading Channels (Wiley, New York, 2005)

11. J Hu, N Beaulieui, Performance analysis of decode-and-forward relaying with selection combining. IEEE Commun Lett. 11(6), 489-491 (2007)

12. M Selvaraj, R Mallik, Error analysis of the decode and forward protocol with selection combining. IEEE Trans Wirel Commun. 8(6), 3086-3094 (2009)

13. M Selvaraj, R Mallik, Scaled selection combining based cooperative diversity system with decode and forward relaying. IEEE Trans Veh Technol. 59(9), 4388-4399 (2010)

14. S Ikki, M Ahmed, Performance analysis of generalized selection combining for decode-and-forward cooperative-diversity networks, in Proceedings of IEEE Vehicular Technology Conference, pp. 1-5 (September 2010)

15. G-T Chyi, J Proakis, C Keller, On the symbol error probability of maximumselection diversity reception schemes over a Rayleigh fading channel. IEEE Trans Commun. 37, 79-83 (1989). doi:10.1109/26.21658 
16. E Neasmith, N Beaulieu, New results on selection diversity. IEEE Trans Commun. 46, 695-704 (1998). doi:10.1109/26.668745

17. R Annavajjala, A Chockalingam, L Milstein, Further results on selection combining of binary NCFSK signals in Rayleigh fading channels. IEEE Trans Commun. 52, 939-952 (2004). doi:10.1109/TCOMM.2004.829530

18. S Haghani, N Beaulieu, M-ary NCFSK with $S+N$ selection combining in Rician fading. IEEE Trans Commun. 54, 491-498 (2006)

19. THimsoon, W Su, K Liu, Differential transmission for amplify-and-forward cooperative communications. IEEE Signal Process Lett. 12, 597-600 (2005)

20. T Himsoon, W Siriwongpairat, W Su, K Liu, Differential modulation with threshold-based decision combining for cooperative communications. IEEE Trans Signal Process. 55, 3905-3923 (2007)

21. Q Zhao, H Li, Differential modulation for cooperative wireless systems. IEEE Trans Signal Process. 55, 2273-2283 (2007)

22. Q Zhao, H Li, P Wang, Performance of cooperative relay with binary modulation in Nakagami- $m$ fading channels. IEEE Trans Veh Technol. 57, 3310-3315 (2008)

23. W Cho, R Cao, L Yang, Optimum resource allocation for amplify-andforward relay networks with differential modulation. IEEE Trans Signal Process. 56, 5680-5691 (2008)

24. W Cho, L Yang, Optimum resource allocation for relay networks with differential modulation. IEEE Trans Commun. 56, 531-534 (2008)

25. D Chen, J Laneman, Modulation and demodulation for cooperative diversity in wireless systems. IEEE Trans Wirel Commun. 5, 1785-1794 (2006)

26. HX Nguyen, $\mathrm{HH}$ Nguyen, Adaptive relaying in noncoherent cooperative networks. IEEE Trans Signal Process. 58, 3938-3945 (2010)

27. DP Bertsekas, Constrained Optimization and Lagrange Multiplier Methods (Athena Scientific, Belmont, 1996)

doi:10.1186/1687-1499-2011-106

Cite this article as: Nguyen and Nguyen: Selection combining for noncoherent decode-and-forward relay networks. EURASIP Journal on Wireless Communications and Networking 2011 2011:106.

\section{Submit your manuscript to a SpringerOpen ${ }^{\mathcal{O}}$ journal and benefit from:}

- Convenient online submission

- Rigorous peer review

- Immediate publication on acceptance

- Open access: articles freely available online

- High visibility within the field

- Retaining the copyright to your article

Submit your next manuscript at $\gg$ springeropen.com 